\title{
MODULI OF SMOOTHNESS AND RATE OF A.E. CONVERGENCE FOR SOME CONVOLUTION OPERATORS
}

\author{
ALEXANDER M. STOKOLOS AND WALTER TREBELS
}

\begin{abstract}
One purpose of this article is to establish results on the rate of almost everywhere convergence of approximation processes of convolution type in $L^{p}\left(\mathbb{R}^{n}\right)$, where instead of a particular rate (like $t^{\mu}, \mu>0, t \rightarrow 0+$ ) fractional moduli of smoothness are employed. An essential tool is a modified $K$-functional. Away from saturation orders these results are nearly optimal. A second purpose is to illustrate that the methods applied also work in other settings which feature a convolution/multiplier structure.
\end{abstract}

\section{INTRODUCTION}

Starting point are the two papers $[21,13]$ on the rate of a.e. convergence of certain integral means of convolution type on $L^{p}(\mathbb{R}), p \geq 1$. One application for the Weierstrass means of the general results there reads as follows.

Let $\varepsilon>0$ and $f \in L^{p}(\mathbb{R}), 1<p<\infty$, satisfy $\sup _{|h|<t}\|f(\cdot+h)-f(\cdot)\|_{p}=$ $O\left(t^{\mu}\right), 0<\mu<1$, then

$$
\frac{1}{2 \pi t} \int_{\mathbb{R}} f(x-y) e^{-(|y| / t)^{2}} d y-f(x)=o_{x}\left(t^{\mu}|\log t|^{1 / p+\varepsilon}\right) \quad \text { a.e. } \quad \text { as } t \rightarrow 0+.
$$

It is impossible to choose $\varepsilon=0$.

Here we considerably generalize the results of [21] in the multivariate framework $L^{p}\left(\mathbb{R}^{n}\right), 1<p<\infty$, by relating the intrinsic smoothness of $f$, given by its norm modulus of smoothness, with the rate of a.e. convergence directly; in particular, moduli of smoothness of fractional order are needed to describe the rate of a.e. convergence for saturated approximation processes like the Riesz or the general Weierstrass/Abel-Cartwright means adequately. Further we indicate that the method employed also works for summability methods of certain orthogonal expansions. The methods of proof themselves were motivated by a programmatic remark due to H. S. Shapiro [17, p. 120]: "Another interesting area for study is how far pointwise (rather than norm) approximation theorems can be inferred from the Fourier transform of the kernel." This means to look at this area from a multiplier point of view.

2010 Mathematics Subject Classification. Primary 41A25; Secondary 41A40, 42B15, 42C10.

Key words and phrases. Moduli of smoothness, Fourier multipliers, $K$-functionals, rate of almost everywhere convergence. 
Here and in the next two sections we deal with the $L^{p}\left(\mathbb{R}^{n}\right)$-setting. First define the modulus of smoothness $\omega_{\lambda}(\cdot, f)_{p}$ of order $\lambda>0$ of $f \in L^{p}, 1<p<\infty$, by

$$
\omega_{\lambda}(t, f)_{p}:=\sup _{|h|<t}\left\|\Delta_{h}^{\lambda} f\right\|_{p}, \quad \Delta_{h}^{\lambda} f(x)=\sum_{j=0}^{\infty}(-1)^{j}\left(\begin{array}{l}
\lambda \\
j
\end{array}\right) f(x+j h), \quad \lambda>0 .
$$

Approximation processes $\left(T_{t} f\right)_{t>0}$ of convolution type on $L^{p}\left(\mathbb{R}^{n}\right), 1<p<\infty$, are considered, where

$$
T_{t} f:=t^{-n} \int_{\mathbb{R}^{n}} K(y / t) f(x-y) d y, \quad K \in L^{1}\left(\mathbb{R}^{n}\right), \quad \int_{\mathbb{R}^{n}} K(y) d y=1 .
$$

It is well-known (see e.g. [18, p.62]) that $\sup _{|y| \geq|x|}|K(y)| \in L^{1}$ implies

$$
\lim _{t \rightarrow 0+} T_{t} f(x)-f(x)=0 \quad \text { a. e. }
$$

Here we are looking for a refinement of this result in the sense of

$$
T_{t} f(x)-f(x)=o(1) \omega_{\lambda}(t, f)_{p} F\left(\omega_{\lambda}(t, f)_{p}\right) \quad \text { a. e. }
$$

Also interesting is the question how the rate of norm convergence directly entails a certain rate of a.e. convergence. The central problem is to find a "good" (decreasing) adjusting function $F:(0,1) \rightarrow(0, \infty)$ ensuring (1.2). It turns out advantageously to replace $\omega_{\lambda}(t, f)$ by Peetre's (modified) $K$-functional. This we define as follows.

Let $\varphi \in \mathcal{S}\left(\mathbb{R}^{n}\right)$ be such that $\operatorname{supp} \varphi=\left\{\xi \in \mathbb{R}^{n}: 1 / 2 \leq|\xi| \leq 2\right\}, \varphi(\xi)>0$ for $1 / 2<|\xi|<2$, and $\sum_{k=-\infty}^{\infty} \varphi_{k}(\xi)=1, \xi \neq 0$, where $\varphi_{k}(\xi)=\varphi\left(2^{-k} \xi\right)$. Then, for $1 \leq p \leq \infty$ and $\lambda>0$, define the Riesz space $H_{\lambda}^{p}\left(\mathbb{R}^{n}\right)$ by (cf. [2, p. 147])

$$
H_{\lambda}^{p}\left(\mathbb{R}^{n}\right):=\left\{f \in L^{p}:\|f\|_{p}+|f|_{H_{\lambda}^{p}}<\infty\right\}, \quad|f|_{H_{\lambda}^{p}}:=\left\|\sum_{k=-\infty}^{\infty} \mathcal{F}^{-1}\left[|\xi|^{\lambda} \varphi_{k}\right] * f\right\|_{p} .
$$

Here $\mathcal{F}$ denotes the Fourier transform $\left(\mathcal{F}^{-1}\right.$ its inverse), defined on the Schwarz test function space $\mathcal{S}\left(\mathbb{R}^{n}\right)$ by

$$
\mathcal{F}[f](\xi) \equiv \widehat{f}(\xi):=\int_{\mathbb{R}^{n}} f(x) e^{-i \xi x} d x, \quad f \in \mathcal{S} .
$$

If one introduces an associated $K$-functional by

$$
K(t, f) \equiv K\left(t, f ; L^{p}, H_{\lambda}^{p}\right):=\inf _{g \in H_{\lambda}^{p}}\left(\|f-g\|_{p}+t|g|_{H_{\lambda}^{p}}\right), \quad t>0,
$$

(note that it is increasing), then Wilmes [29] has shown that

$$
K\left(t^{\lambda}, f ; L^{p}\left(\mathbb{R}^{n}\right), H_{\lambda}^{p}\right) \approx \omega_{\lambda}(t, f)_{p}, \quad 1<p<\infty, \lambda>0 .
$$

Observing that for $\lambda=N \in \mathbb{N}$ and $1<p<\infty$ the Riesz space $H_{N}^{p}$ can be identified with the Sobolev space $W_{N}^{p}: H_{N}^{p}=W_{N}^{p}$, Wilmes' result is not surprising in view of the well-known characterization [1, p. 341]. 
In the following we will only consider approximation processes of type (1.1) with radial kernels, i.e., $K(y):=k(|y|)$. Since $\widehat{K}$ is also radial, we write $\widehat{K}(\xi):=$ $m(|\xi|)$. Thus the approximation processes to be discussed may be written in the form

$$
T_{m_{t}} f=\mathcal{F}^{-1}[m(t|\xi|)] * f, \quad \mathcal{F}^{-1}[m(|\xi|)] \in L^{1}\left(\mathbb{R}^{n}\right), \quad m(0)=1 .
$$

Also we restrict ourselves to two cases: the saturated means which satisfy

$$
\lim _{s \rightarrow 0+} \frac{1-m(s)}{s^{\gamma}}=c, \quad c \neq 0, c \in \mathbb{C}, \text { some } \gamma>0,
$$

and the non-saturated ones for which $c=0$ for all $\gamma>0$. This automatically leads to the Riesz spaces $H_{\gamma}^{p}, \gamma>0$ as saturation classes. Typical examples of saturated processes with saturation class $H_{\gamma}^{p}$ are the general Riesz means $\left(R_{t}^{\delta, \gamma}\right)_{t>o}$ and the general Weierstrass means $\left(W_{t}^{\gamma}\right)_{t>o}$. In the first case there is $m_{\delta, \gamma}(u)=\left(1-u^{\gamma}\right)_{+}^{\delta}$, where $\gamma>0$ and $\delta>(n-1) / 2$, in the second $m_{\gamma}(u)=$ $e^{-u^{\gamma}}, \gamma>0$. Both means can be used [25] to characterize the $K$-functional

$$
K\left(t^{\gamma}, f ; L^{p}\left(\mathbb{R}^{n}\right), H_{\gamma}^{p}\right) \approx\left\|R_{t}^{\delta, \gamma} f-f\right\|_{p} \approx\left\|W_{t}^{\gamma} f-f\right\|_{p}, \quad 1 \leq p<\infty .
$$

Before formulating a first result we quote two lemmas concerning multipliers useful when estimating norms of convolution operators and maximal functions. By purpose, we restrict ourselves to derivatives of integer order - for fractional derivatives see the corresponding references. We employ the following notation $A \approx B$ if $A \lesssim B$ and $A \gtrsim B$, where the former (the latter) is the shortcut of $A \leq c B(A \geq c B)$ for some constant $c>0$ independent of essential quantities.

Lemma 1.1. [24] Let $m$ be a measurable, bounded, sufficiently smooth function on $(0,+\infty)$ which vanishes at infinity and which satisfies for some integer $N>$ $(n-1) / 2$

$$
A_{N}(m) \equiv \int_{0}^{\infty} u^{N}\left|m^{(N+1)}(u)\right| d u<\infty .
$$

Then $\mathcal{F}^{-1}[m(|\xi|)] \in L^{1}\left(\mathbb{R}^{n}\right)$ and $\left\|\mathcal{F}^{-1}[m]\right\|_{1} \lesssim A_{N}(m)$.

Lemma 1.2. $[5,6]$ Let $m$ be a measurable, bounded, sufficiently smooth function on $(0,+\infty)$ which vanishes at infinity and which satisfies for some $N \in \mathbb{N}, N>n|1 / p-1 / 2|+1 / 2$,

$$
B_{N}(m) \equiv\left(\int_{0}^{\infty}\left|u^{N} m^{(N)}(u)\right|^{2} \frac{d u}{u}\right)^{1 / 2}+\int_{0}^{\infty} u^{N-1}\left|m^{(N)}(u)\right| d u<\infty .
$$

Define the maximal operator $T_{m}^{*}$ on $L^{2}\left(\mathbb{R}^{n}\right)$ by

$$
T_{m}^{*} f(x)=\sup _{t>0}\left|T_{m_{t}} f(x)\right|, \quad T_{m_{t}} f(x)=\mathcal{F}^{-1}[m(t|\xi|) \widehat{f}](x) .
$$

Then $T_{m}^{*}$ is of strong type $(p, p), 1<p<\infty$, with the operator norm estimate $\left\|T_{m}^{*}\right\|_{p \rightarrow p} \lesssim B_{N}(m)$; also $T_{m}^{*}$ is of weak type $(1,1)$. 
Our main result concerning the problem (1.2) reads as follows.

Theorem 1.3. Let $f \in L^{p}\left(\mathbb{R}^{n}\right), 1<p<\infty$. For $\lambda>0$, choose a sequence $\left(\theta_{\ell}\right)_{\ell \in \mathbb{N}_{0}}$ via $K\left(\theta_{\ell}^{\lambda}, f\right) \equiv K\left(\theta_{\ell}^{\lambda}, f ; L^{p}\left(\mathbb{R}^{n}\right), H_{\lambda}^{p}\left(\mathbb{R}^{n}\right)\right)=2^{-\ell}$, and a decreasing function $F:(0,1) \rightarrow(0, \infty)$ with $\sum_{\ell=0}^{\infty} 1 / F^{p}\left(2^{-\ell}\right)<\infty$. Further assume that the function $t F(t)$ is increasing. Let $\psi \geq 0$ be a smooth cut-off function with $\psi(t)=1$ if $0 \leq t \leq 1 / 2$, and $=0$ if $t \geq 3 / 4$. Let $h \geq 0$ be a bump function with supp $h \subset[1 / 2,2]$ such that $\sum_{j=0}^{\infty} h\left(2^{j} s\right)=1,0<s<1$, and let $m$ be sufficiently smooth with finite

$B_{N}(m(1-\psi)), \quad B_{N}\left(\left(1-m\left(2^{-j} \cdot\right)\right) h\right)=: B_{j}, \quad j \in \mathbb{N}_{0}, \quad N \in \mathbb{N}, \quad N \geq(n+1) / 2$.

(a) If

$$
\sum_{j=0}^{\infty} B_{j} 2^{j \lambda}\left(\sum_{\ell: \theta_{\ell}<2^{-j}}^{\infty}\left(\frac{1}{F\left(2^{-\ell-1}\right)}\right)^{p}\right)^{1 / p}<\infty
$$

then we have

$$
\mathcal{F}^{-1}[m(t|\xi|)] * f(x)-f(x)=o_{x}(1) K\left(t^{\lambda}, f\right) F\left(K\left(t^{\lambda}, f\right)\right) \text { a. e., } \quad t \rightarrow 0+,
$$

where $o_{x}(1) \in L^{p}\left(\mathbb{R}^{n}\right)$.

(b) If $f$ additionally satisfies $K\left(t^{\lambda}, f\right) \lesssim t^{\eta}$ for some fixed $\eta, 0<\eta<\lambda$, and $F(t):=1+|\log t|^{1+1 / p+\varepsilon}, \varepsilon>0$, with $\sum_{j=0}^{\infty}(j+1)^{-1-\varepsilon} B_{j} 2^{j \lambda}<\infty, \quad \varepsilon>0$, then, for $t \rightarrow 0+$,

$$
\mathcal{F}^{-1}[m(t|\xi|)] * f(x)-f(x)=o_{x}(1) K\left(t^{\lambda}, f\right)\left|\log K\left(t^{\lambda}, f\right)\right|^{1+1 / p+\varepsilon} \text { a. e., }
$$

where again $o_{x}(1) \in L^{p}\left(\mathbb{R}^{n}\right)$.

In view of (1.4), the assumption $K\left(t^{\lambda}, f\right) \lesssim t^{\eta}$ means that $f$ should have some Hölder smoothness.

Examples 1.4. (a) Standard choices for the adjusting function $F$ are $F(t)=$ $1+|\log t|^{1 / p+\varepsilon}, 0<t<1$, or $F(t)=1+|\log t|^{1 / p}|\log | \log t||^{1 / p+\varepsilon}$, where $0<t<$ $1 / 2, \varepsilon>0$, etc.; in these cases, certainly $t F(t)$ is increasing.

(b) We start by giving two examples of non-saturated approximation processes.

(i) DE La Vallée Poussin means. Consider a $C^{\infty}$-cut-off-function $m$ : $[0, \infty) \rightarrow[0,1], m(u)=1$ for $0 \leq u \leq 1$ and $m(u)=0$ for $2 \leq u<\infty$. By Lemma 1 it is clear that $m(|\xi|)$ is the Fourier transform of an admissible approximation kernel. Further, condition (1.6) is satisfied. Now define $V_{t} f:=\mathcal{F}^{-1}[m(t|\xi|)] * f$ as de La Vallée Poussin means and observe that, by Lemma $2, B_{j}=0$ for $j \geq 2$, the remaining $B$ 's are finite. Thus, by the characterization of the $K$-functional in [1, p. 341], we obtain for any fixed $k \in \mathbb{N}, \varepsilon>0$,

$$
V_{t} f(x)-f(x)=o_{x, k}(1) \omega_{k}(t, f)_{p}\left|\log \omega_{k}(t, f)_{p}\right|^{1 / p+\varepsilon} \quad \text { a.e. }, \quad t \rightarrow 0+.
$$


For comparison reasons we note the associated norm convergence result

$$
\left\|V_{t} f-f\right\|_{p} \leq C_{k} \omega_{k}(t, f)_{p}, \quad f \in L^{p}, \quad k \in \mathbb{N} .
$$

(ii) Choose $m:(0, \infty) \rightarrow[0,1], m(u)=1-e^{-1 / u}, m(0)=1$. Since $m \in C^{\infty}$ and $\lim _{u \rightarrow \infty} m(u)=0$, by Lemma 1 we have $\mathcal{F}^{-1}[m(|\xi|)] \in L^{1}\left(\mathbb{R}^{n}\right)$. Further, $B_{N}\left(\left(1-e^{-1 / t}\right)(1-\psi(t))<\infty\right.$ and $B_{j}=O\left(2^{j} e^{-2^{j-1}}\right), j \in \mathbb{N}_{0}$, so that by Lemma 2 we see that, as in the previous example, we have for any fixed $k \in \mathbb{N}$ and $t \rightarrow 0+$

$$
\mathcal{F}^{-1}\left[1-e^{-1 /(t|\xi|)}\right] * f(x)-f(x)=o_{x, k}(1) \omega_{k}(t, f)_{p}\left|\log \omega_{k}(t, f)_{p}\right|^{1 / p+\varepsilon} \quad \text { a.e. }
$$

As already mentioned, typical examples of saturated approximation processes are the general Riesz means, the general Weierstrass means, but also the integral means occuring in Lebesgue's differentiation theorem. Theorem 1.3 now yields the following results.

(iii) General Riesz means. Consider the general Riesz means from (1.7), i.e., $m(u) \equiv m_{\delta, \gamma}(u)=\left(1-u^{\gamma}\right)_{+}^{\delta}$.. Then the assumptions of Theorem 1.3 (a) are satisfied, since $B_{j}=O\left(2^{-j \gamma}\right)$ and $B_{N}(m(u)(1-\psi))<\infty$ when $0<\lambda<\gamma$. Thus, we get with $F(t)=|\log t|^{1 / p+\varepsilon}$ that

$$
R_{t}^{\delta, \gamma} f(x)-f(x)=o_{x, \lambda, \gamma}(1) \omega_{\lambda}(t, f)_{p}\left|\log \omega_{\lambda}(t, f)_{p}\right|^{1 / p+\varepsilon} \quad \text { a.e. }, \quad t \rightarrow 0+.
$$

In view of (1.4) and (1.7) the associated norm convergence result reads

$$
\left\|R_{t}^{\delta, \gamma} f-f\right\|_{p} \approx \omega_{\gamma}(t, f)_{p}, \quad f \in L^{p} .
$$

When we choose $F(t)=|\log t|^{1+1 / p+\varepsilon}$, then the hypotheses of Theorem 1.3 (b) are satisfied even when $\lambda=\gamma$ provided the additional smoothness assumption $\omega_{\gamma}(t, f)_{p} \lesssim t^{\eta}$ for some $\eta, 0<\eta<\gamma$, holds. Therefore, we have for almost all $x \in \mathbb{R}^{n}$ that

$$
R_{t}^{\delta, \gamma} f(x)-f(x)=o_{x}(1) \omega_{\gamma}(t, f)_{p}\left|\log \omega_{\gamma}(t, f)_{p}\right|^{1+1 / p+\varepsilon} \quad \text { a.e. }, \quad t \rightarrow 0+,
$$

or, when inferring pointwise convergence from strong convergence,

$$
R_{t}^{\delta, \gamma} f(x)-f(x)=o_{x}(1)\left\|R_{t}^{\delta, \gamma} f-f\right\|_{p}\left|\log \left\|R_{t}^{\delta, \gamma} f-f\right\|_{p}\right|^{1+1 / p+\varepsilon} \text { a.e., } t \rightarrow 0+.
$$

We point out that, probably caused by the method of proof, the above result involving $\omega_{\gamma}(t, f)_{p}$ does not look natural "near" the saturation order $t^{\gamma}$ (like $\left.t^{\gamma}|\log t|\right)$, since in the saturation case $R_{t}^{\delta, \gamma} f(x)-f(x)=O_{x}\left(t^{\gamma}\right)-$ see [21].

(iv) General Weierstrass means. In this case the choice $m_{\gamma}(u)=e^{-u^{\gamma}}$ yields the same approximation results as in (iii) for the the general Riesz means.

(v) Lebesgue's Differentiation Theorem. This can also be looked at under the aspect of the rate of a.e.-convergence. Observe that (cf. [19, p. 171]

$$
\frac{1}{\left|B_{t}(0)\right|} \int_{B_{t}(0)} f(x+y) d y=\mathcal{F}^{-1}[m(t|\xi|)] * f(x), \quad m(u)=c_{n} u^{-n / 2} J_{n / 2}(u),
$$


where $J_{\alpha}$ denotes the Bessel function of order $\alpha$ and $c_{n}$ is given by $m(0) 1$. Clearly, $T_{m(1-\psi)}^{*}$ essentially is the Hardy-Littlewood maximal function, thus $\left\|T_{m(1-\psi)}^{*}\right\|_{p \rightarrow p}<\infty$, which replaces the condition $B_{N}(m(1-\psi))<\infty$ in Theorem 1.3. Using the power series representation of the Bessel function it immediately follows that $B_{j}=O\left(2^{-2 j}\right)$. Hence, for $0<\lambda<2$, we obtain almost everywhere

$$
\frac{1}{\left|B_{t}(0)\right|} \int_{B_{t}(0)} f(x+y) d y=f(x)+o_{x}(1) \omega_{\lambda}(t, f)_{p}\left|\log \omega_{\lambda}(t, f)_{p}\right|^{1 / p+\varepsilon}, \quad t \rightarrow 0+,
$$

or, when $f$ satisfies a Hölder condition in $L^{p}$-norm, we get for $t \rightarrow 0+$

$$
\frac{1}{\left|B_{t}(0)\right|} \int_{B_{t}(0)} f(x+y) d y=f(x)+o_{x}(1) \omega_{2}(t, f)_{p}\left|\log \omega_{2}(t, f)_{p}\right|^{1+1 / p+\varepsilon} \quad \text { a.e.. }
$$

\section{Proof of Theorem 1.3.}

Consider a smooth cut-off function $\psi$ with $\psi(u)=1$ for $u \leq 1 / 2$ and $=0$ for $u \geq 3 / 4$. Then

$$
T_{m_{t}} f-f=T_{\left(m_{t}-1\right) \psi_{t}} f+T_{\left(m_{t}-1\right)\left(1-\psi_{t}\right)} f .
$$

First consider the contribution given by the multiplier $(m-1)(1-\psi)$. By the choice of $\psi$ we have

$$
\operatorname{supp}(m-1)(1-\psi) \subset\{\xi:|\xi| \geq 1 / 2\} .
$$

Introduce $\chi \in C_{0}^{\infty}$ such that $\chi(\xi)=1$ for $|\xi| \leq 1 / 2$ and $\chi(\xi)=0$ for $|\xi| \geq 1$, then

$$
\left\|T_{1-\chi_{t}} f\right\|_{p} \leq\left\|T_{\chi_{t}} f-f\right\|_{p} \lesssim K\left(t^{\lambda}, f\right) \quad 0<t<1 .
$$

This follows from the standard estimate: For all $g \in S$ there holds

$$
\left\|T_{1-\chi_{t}} f\right\|_{p} \leq\left\|T_{1-\chi_{t}}(f-g)\right\|_{p}+\left\|T_{\chi_{t}} g-g\right\|_{p} \lesssim\|f-g\|_{p}+t^{\lambda}\left\|\mathcal{F}^{-1}\left[|\xi|^{\lambda} \widehat{g}\right]\right\|_{p}
$$

and hence also for the infimum over all $g \in S$. Set

$$
w(t):=K\left(t^{\lambda}, f\right) F\left(K\left(t^{\lambda}, f\right)\right)
$$

and assume $\theta_{\ell+1}<t \leq \theta_{\ell}$. Since

$$
\operatorname{supp}\left(m_{t}-1\right)\left(1-\psi_{t}\right) \subset\left\{\xi:|\xi| \geq \frac{1}{2 \theta_{\ell}}\right\}, \quad 1-\chi_{2 \theta_{\ell}}(\xi)=1 \text { for }|\xi| \geq \frac{1}{2 \theta_{\ell}},
$$

we have

$$
T_{\left(m_{t}-1\right)\left(1-\psi_{t}\right)} f=T_{\left(m_{t}-1\right)\left(1-\psi_{t}\right)\left(1-\chi_{2 \theta_{\ell}}\right)} f=T_{\left(m_{t}-1\right)\left(1-\psi_{t}\right)}\left(T_{1-\chi_{2 \theta_{\ell}}} f\right) .
$$

This implies the key relation

$$
\left|T_{\left(m_{t}-1\right)\left(1-\psi_{t}\right)} f(x)\right| \leq C \sup _{\ell \geq 0} \frac{T_{(m-1)(1-\psi)}^{*}\left(T_{1-\chi_{2 \theta_{\ell}}} f\right)(x)}{w\left(\theta_{\ell+1}\right)} w(t), \quad t>0,
$$

for almost all $x \in \mathbb{R}^{n}$. But

$$
T_{\left(m_{t}-1\right)\left(1-\psi_{t}\right)} f=T_{m_{t}\left(1-\psi_{t}\right)} f+T_{\psi_{t}} f-f,
$$


So

$$
T_{(m-1)(1-\psi)}^{*} f \leq T_{m(1-\psi)}^{*} f+T_{\psi}^{*} f+|f| .
$$

Now $T_{\psi}^{*}, T_{m(1-\psi)}^{*}: L^{p} \rightarrow L^{p}$ are bounded maximal operators by Lemma 1.2 and the hypothesis. So we may conclude that $\left\|T_{(m-1)(1-\psi)}^{*} \mid\right\|_{p \rightarrow p}<\infty$ and, therefore,

$$
\begin{aligned}
\left\|\sup _{\ell \geq 0} \frac{T_{(m-1)(1-\psi)}^{*}\left(T_{1-\chi_{2 \theta_{\ell}}} f\right)}{w\left(\theta_{\ell+1}\right)}\right\|_{p}^{p} & \lesssim \sum_{\ell \geq 0} \frac{\left\|T_{(m-1)(1-\psi)}^{*}\left(T_{1-\chi_{2 \theta_{\ell}}} f\right)\right\|_{p}^{p}}{w\left(\theta_{\ell+1}\right)^{p}} \\
& \lesssim\left\|T_{(m-1)(1-\psi)}^{*}\right\|_{p \rightarrow p}^{p} \sum_{\ell \geq 0} \frac{\left\|T_{1-\chi_{2 \theta_{\ell}}} f\right\|_{p}^{p}}{w\left(\theta_{\ell+1}\right)^{p}} .
\end{aligned}
$$

The estimate (2.2) and the definition of $w(t)$ give

$$
\left\|\sup _{\ell \geq 0} \frac{T_{(m-1)(1-\psi)}^{*}\left(T_{1-\chi_{2 \theta_{\ell}}} f\right)}{w\left(\theta_{\ell+1}\right)}\right\|_{p}^{p} \leq C_{p}(m, \psi) \sum_{\ell=1}^{\infty}\left(\frac{1}{F\left(K\left(\theta_{\ell}^{\lambda}, f\right)\right)}\right)^{p}
$$

which is finite by hypothesis (1.9) (only the contribution $j=0$ is needed here).

Now we estimate the contribution caused by $T_{\left(m_{t}-1\right) \psi_{t}} f(x)$.

Set $\mu \equiv(m-1) \psi$ and remember that $\mu=0$ for $|\xi| \geq 3 / 4$ and $\mu(\xi)=m(\xi)-1$ for $|\xi| \leq 1 / 2$. Recall the partition of unity from the assumptions of Theorem 1.3

$$
\sum_{j=0}^{\infty} h\left(2^{j} t|\xi|\right)=1 \quad \text { for } \quad|t \xi|<1, \quad 0<t<1 .
$$

Without loss of generality we may assume $\theta_{0}=1$ and choose $\ell_{0}=\ell_{0}(j) \in \mathbb{N}$ such that $\theta_{\ell_{0}} \geq 2^{-j}$ but $\theta_{\ell_{0}+1}<2^{-j}$. Then

$$
\begin{aligned}
\sup _{0<t<1} \frac{\left|T_{\mu_{t}} f\right|}{w(t)} & \leq \sum_{j=0}^{\infty} \sup _{0<t<1} \frac{\left|T_{\mu_{t} h_{t 2^{j}}} f\right|}{w(t)} \\
& \leq \sum_{j=0}^{\infty} \sup _{\ell \geq \ell_{0}} \sup _{\theta_{\ell+1}<t \leq \theta_{\ell}} \frac{\left|T_{\mu_{t}} h_{t 2^{j}} f\right|}{w(t)}+\sum_{j=0}^{\infty} \sup _{2^{-j}<t \leq 1} \frac{\left|T_{\mu_{t}} h_{t 2^{j}} f\right|}{w\left(2^{-j}\right)} .
\end{aligned}
$$

In the last term we used that $w$ is increasing. We begin to discuss the critical first sum on the right side of (2.5). It is clear that $\theta_{\ell+1}<t \leq \theta_{\ell} \operatorname{implies} \operatorname{supp}\left(h_{t 2^{j}}\right) \subset$ $\left[\left(2^{j+1} \theta_{\ell}\right)^{-1},\left(2^{j-1} \theta_{\ell+1}\right)^{-1}\right]$. Now choose a non-decreasing function $\Phi \in C^{\infty}[0, \infty)$ such that $\Phi(|\xi|)=1$ for $|\xi| \geq 1 / 2$ and $\Phi(|\xi|)=0$ for $|\xi| \leq 1 / 4$ and set $\Phi_{\ell, j}(|\xi|)=$ $\Phi\left(2^{j} \theta_{\ell}|\xi|\right)$. Hence $\Phi_{\ell, j}(|\xi|)=1$ for $|\xi| \geq\left(2^{j+1} \theta_{\ell}\right)^{-1}$ and, therefore, we have 
$\Phi_{\ell, j} h_{t 2^{j}}=h_{t 2^{j}}$ provided $\theta_{\ell+1}<t \leq \theta_{\ell}$. Thus

$$
\begin{aligned}
\left\|\sum_{j=0}^{\infty} \sup _{\ell \geq \ell_{0}} \frac{\sup _{\theta_{\ell+1}<t \leq \theta_{\ell}}\left|T_{\mu_{t} h_{t 2}} f\right|}{w\left(\theta_{\ell+1}\right)}\right\|_{p} & \leq \sum_{j=0}^{\infty}\left\|\sup _{\ell \geq \ell_{0}} \frac{T_{\mu h_{2^{j}}}^{*}\left(T_{\Phi_{\ell, j}} f\right)}{w\left(\theta_{\ell+1}\right)}\right\|_{p} \\
& \leq \sum_{j=0}^{\infty}\left(\sum_{\ell \geq \ell_{0}}\left(\frac{\left\|T_{\mu h_{2^{j}}}^{*}\left(T_{\Phi_{\ell, j}} f\right)\right\|_{p}}{w\left(\theta_{\ell+1}\right)}\right)^{p}\right)^{1 / p} \\
& \leq \sum_{j=0}^{\infty}\left\|T_{\mu h_{2^{j}}}^{*}\right\|_{p \rightarrow p}\left(\sum_{\ell \geq \ell_{0}}\left(\frac{\left\|T_{\Phi_{\ell, j}} f\right\|_{p}}{w\left(\theta_{\ell+1}\right)}\right)^{p}\right)^{1 / p}
\end{aligned}
$$

Now observe that $\left\|T_{\Phi_{\ell, j}} f\right\|_{p}=\left\|T_{1-\Phi_{\ell, j}} f-f\right\|_{p} \lesssim K\left(\left(2^{j} \theta_{\ell}\right)^{\lambda}, f\right)$ analogous to (2.2). Therefore, we can continue the preceding estimate by

$$
\begin{aligned}
& \lesssim \sum_{j=0}^{\infty}\left\|T_{\mu h_{2 j}}^{*}\right\|_{p \rightarrow p}\left(\sum_{\ell \geq \ell_{0}}\left(\frac{K\left(\left(2^{j} \theta_{\ell}\right)^{\lambda}, f\right)}{w\left(\theta_{\ell+1}\right)}\right)^{p}\right)^{1 / p} \\
& \lesssim \sum_{j=0}^{\infty} B_{j} 2^{j \lambda}\left(\sum_{\ell \geq \ell_{0}}\left(\frac{K\left(\theta_{\ell}^{\lambda}, f\right)}{w\left(\theta_{\ell+1}\right)}\right)^{p}\right)^{1 / p} \lesssim \sum_{j=0}^{\infty} B_{j} 2^{j \lambda}\left(\sum_{\ell \geq \ell_{0}}\left(\frac{1}{F\left(2^{-\ell}\right)}\right)^{p}\right)^{1 / p}
\end{aligned}
$$

For the last estimates we used the dilation invariance of the integral conditions in (1.8), namely $B_{N}\left(m_{t}\right)=B_{N}(m), t>0$, and $w\left(\theta_{\ell+1}\right) \approx 2^{-\ell \lambda} F\left(2^{-\ell}\right)$.

The second series on the right side of $(2.5)$ is even easier to estimate, since

$$
\begin{aligned}
\left\|\sum_{j=0}^{\infty} \sup _{2^{-j}<t<1} \frac{\left|T_{\mu_{t} h_{t 2 j}} f\right|}{w\left(2^{-j}\right)}\right\|_{p} & \lesssim \sum_{j=0}^{\infty}\left\|T_{\mu h_{2 j}}^{*}\right\|_{p \rightarrow p}\|f\|_{p} \frac{1}{w\left(2^{-j}\right)} \\
& \lesssim \sum_{j=0}^{\infty} B_{j} 2^{j \lambda} \frac{\|f\|_{p}}{F\left(2^{-j \lambda}\right)}
\end{aligned}
$$

which is finite by (1.9) (for the simplification of the denominator we used that $\left.K\left(2^{-j \lambda}, f\right) \geq 2^{-j \lambda} K(1, f)\right)$.

Summarizing, in view of (2.1), we see that by the hypothesis (1.9) the estimates (2.5), (2.6) (clearly $\ell_{0} \geq 0$ ) in combination with (2.2), (2.4) show that

$$
\sup _{0<t<1} \frac{T_{m_{t}} f(x)-f(x)}{K\left(t^{\lambda}, f\right) F\left(K\left(t^{\lambda}, f\right)\right)}<\infty \quad \text { a.e., } t \rightarrow 0+,
$$

which proves Theorem 1.3 (a).

Concerning Part (b) observe that, in view of the above proof, we have only to estimate the last double sum in (2.6) conveniently. By the the smoothness of $f$ the choice of $\theta_{\ell_{0}(j)}$ yields $\ell_{0}(j) \gtrsim j+1$ since

$$
2^{-\ell_{0}(j)}=K\left(\theta_{\ell_{0}(j)}^{\lambda}, f\right)=2 K\left(\theta_{\ell_{0}(j)+1}^{\lambda}, f\right) \lesssim \theta_{\ell_{0}(j)+1}^{\eta}<2^{-j \eta} .
$$


Therefore, with $F(t)=|\log t|^{1+1 / p+\varepsilon}$,

$$
\left(\sum_{\ell \geq \ell_{0}}\left(\frac{1}{F\left(2^{-\ell}\right)}\right)^{p}\right)^{1 / p} \lesssim\left(\sum_{\ell \geq \ell_{0}} \ell^{-p-1-\varepsilon p}\right)^{1 / p} \lesssim \ell_{0}^{-1-\varepsilon} \lesssim(j+1)^{-1-\varepsilon},
$$

which gives the assertion (b).

Remark 2.1. (a) On account of [8, Theorem 3.14] the results in Section 1 also hold in the framework of Lorentz-Zygmund spaces discussed there.

(b) Further, these results easily carry over to the $L^{p}\left(\mathbb{T}^{n}\right)$-setting, $1<p<\infty$, in view of the results in [19, Chap. VII, §3], [14], and [30].

\section{Generalizations}

We want to show that the above method to obtain rates for a.e. convergence also works in other settings. For this purpose note that recalling the methods of Section 2 one realizes that we have only needed control over norms of operators given via multipliers, some norm estimates for the rate of convergence of de La Vallée Poussin type means via the $K$-functional and characterizations of the $K$-functional. Concerning a characterization via moduli of smoothness based on (generalized) translations we have needed the special structure of $\mathbb{R}^{n}$. Apart from that $\mathbb{R}^{n}$ did not appear once one has the required multiplier criteria.

We start by briefly describing the abstract setting concerning eigenfunction expansions given in [4]. For a Lebesgue measurable set $E \subset \mathbb{R}^{n}$ and a nonnegative measure $\mu$ on $E$ define the space $L^{p}(E, d \mu), 1<p<\infty$, as the Banach space of all (Lebesgue) measurable functions on $E$ with finite norm

$$
\|f\|_{p}:=\left(\int_{E}|f(x)|^{p} d \mu(x)\right)^{1 / p}
$$

Assume that there exists a sequence of projections $\left(P_{k}\right)_{k \in \mathbb{N}_{0}}, P_{k}: L^{p} \rightarrow L^{p}$, being linear, bounded, and satisfying

(i) $P_{j} P_{k}=\delta_{j, k} P_{k}, \delta_{j, k}$ being Kronecker's symbol (mutual orthogonality);

(ii) the sequence $\left(P_{k}\right)$ is total, i.e., $P_{k} f=0$ for all $k \in \mathbb{N}_{0}$ implies $f=0$;

(iii) the sequence $\left(P_{k}\right)$ is fundamental, i.e., the linear span of the ranges $P_{k}\left(L^{p}\right)$ is dense: $\overline{\bigcup_{k \in \mathbb{N}_{0}} P_{k}\left(L^{p}\right)}=L^{p}$.

Then, with each $f \in L^{p}$ one may associate its (formal) Fourier series expansion $f \sim \sum_{k=0}^{\infty} P_{k} f$. Denote by $\mathfrak{P} \subset L^{p}$ the set of all "polynomials", i.e., those $f \in X$, for which only finitely many $P_{k} f \neq 0$. Given a scalar-valued sequence $m=$ $\left(m_{k}\right)_{k \in \mathbb{N}_{0}}$, we define the linear operator $T_{m}: \mathfrak{P} \rightarrow \mathfrak{P}$ by $T_{m} f=\sum_{k=0}^{\infty} m_{k} P_{k} f$. 
In particular, employing the notation $\phi_{c}^{\gamma}:[0, \infty) \rightarrow \mathbb{R}, \phi_{c}^{\gamma}(u)=(u(u+c))^{\gamma / 2}$, we define an abstract derivative $D_{c}^{\gamma}: D_{p}\left(D_{c}^{\gamma}\right) \rightarrow L^{p}$, first on $\mathfrak{P}$ by

$$
D_{c}^{\gamma} f:=\sum_{k=0}^{\infty} \phi_{c}^{\gamma}(k) P_{k} f, \quad \gamma>0, \quad c \geq 0, \quad f \in \mathfrak{P},
$$

and then take its closure in $L^{p}$, i.e., to each $f \in D_{p}\left(D_{c}^{\gamma}\right)$ there exist $f_{j} \in \mathfrak{P}$ with $\left\|f-f_{j}\right\|_{p} \rightarrow 0$ and $\left(D_{c}^{\gamma} f_{j}\right)_{j}$ is a Cauchy sequence in $L^{p}$. We call $m$ a multiplier on $L^{p}$, notation $m \in M^{p}$, if

$$
\|m\|_{M}:=\sup \left\{\left\|T_{m} f\right\|_{p}:\|f\|_{p} \leq 1, f \in L^{p}\right\}
$$

is finite (on account of (iii) there is a unique extension of $T_{m}$ from $\mathfrak{P}$ to $L^{p}$ ).

To ensure control over maximal functions, which were essentially used in the $L^{p}\left(\mathbb{R}^{n}\right)$-case, we assume for some $N \in \mathbb{N}_{0}$ the following property

$$
\left\|\sup _{k}(C, N)_{k} f\right\|_{p} \leq C_{N}\|f\|_{p} \text { for all } f \in L^{p}, 1<p<\infty
$$

where the $(C, N)_{k} f$ denote the Cesàro means of order $N$ of $f \in L^{p}$. These are given by

$$
(C, N)_{k} f:=\left(A_{k}^{N}\right)^{-1} \sum_{i=0}^{k} A_{k-i}^{N} P_{i} f, \quad A_{k}^{N}=\left(\begin{array}{c}
k+N \\
k
\end{array}\right) .
$$

Clearly, (3.1) implies the boundedness of the Cesàro means

$$
\left\|(C, N)_{k} f\right\|_{p} \leq C_{N}\|f\|_{p} \text { for all } f \in L^{p}, 1<p<\infty,
$$

which immediately leads to a substitute of Lemma 1.1 - for the idea of the proof see the proof of Lemma 4.2 below (cf. [4, II]).

Lemma 3.1. (a) Let the projections $\left(P_{k}\right)_{k \in \mathbb{N}_{0}} \subset \mathcal{L}\left(L^{p}\right)$ be as above and (3.3) hold. Then

$$
\left\|T_{m} f\right\| \leq C_{N} \sum_{k=0}^{\infty}\left(\begin{array}{c}
k+N \\
k
\end{array}\right)\left|\Delta^{N+1} m_{k}\right|\|f\|_{p} \quad \text { for all } f \in L^{p}
$$

(b) If one defines for continuous $m:[0, \infty) \rightarrow \mathbb{C}$ with $\lim _{t \rightarrow \infty} m(t)=0$ an operator $T_{m_{\phi ; t}}$ via the family of sequences $m_{\phi ; t}:=\left(m\left(\rho(t) \phi_{c}^{\gamma}(k)\right)\right)_{k \in \mathbb{N}_{0}}, \gamma>0$, with $\rho:[0, \infty) \rightarrow[0, \infty), \rho(0)=0$, strictly increasing then, for sufficiently smooth $m$,

uniformly in $t$.

$$
\left\|T_{m_{\phi ;}} f\right\|_{p} \lesssim \int_{0}^{\infty} s^{N}\left|m^{(N+1)}(s)\right| d s\|f\|_{p}
$$

The latter estimate follows by a variant of the Second Theorem of Consistency - see [23, Lemma 3.8]. Recall $\phi_{c}^{\gamma}(u)=(u(u+c))^{\gamma / 2}$ and note that $\rho(t)$ will, e.g., be specialized to $2^{j} \phi_{c}^{\gamma}(t)$.

One substitute of Lemma 1.2 reads as follows. 
Lemma 3.2. Let the projections $\left(P_{k}\right)_{k \in \mathbb{N}_{0}} \subset \mathcal{L}\left(L^{p}\right)$ be as above, let (3.1) hold, and $m$ be as in Lemma 3.1 (b). Define the maximal operator $T_{m_{\phi}}^{*}$ by

$$
T_{m_{\phi}}^{*} f:=\sup _{t>0}\left|T_{m_{\phi ; t}} f\right| .
$$

Then, for sufficiently smooth $m$ and all $f \in L^{p}$,

$$
\left\|T_{m_{\phi}}^{*} f\right\|_{p} \lesssim B_{N}^{*}(m)\|f\|_{p}, \quad B_{N}^{*}(m):=\int_{0}^{\infty} s^{N}\left|m^{(N+1)}(s)\right| d s .
$$

In the special case $\rho(t)=\phi_{c}^{\gamma}(t), c=0, \gamma=1$, the maximal function is described on $\mathfrak{P}$ by $\sup _{t>0}\left|\sum_{k=0}^{\infty} m(t k) P_{k} f\right|$.

Proof. As is well-known (cf. Part II of [4])

$$
\begin{aligned}
\left|T_{m_{\phi ;}} f\right| & =\left|\sum_{k=0}^{\infty}\left(\begin{array}{c}
k+N \\
k
\end{array}\right) \Delta^{N+1} m\left(\rho(t) \phi_{c}^{\gamma}(k)\right)(C, N)_{k} f\right| \\
& \leq\left|\sup _{k}(C, N)_{k} f\right| \sum_{k=0}^{\infty}\left(\begin{array}{c}
k+N \\
k
\end{array}\right)\left|\Delta^{N+1} m\left(\rho(t) \phi_{c}^{\gamma}(k)\right)\right| \\
& \lesssim\left|\sup _{k}(C, N)_{k} f\right| \int_{0}^{\infty} s^{N}\left|(d / d s)^{(N+1)} m\left(\rho(t) \phi_{c}^{\gamma}(s)\right)\right| d s \\
& \lesssim\left|\sup _{k}(C, N)_{k} f\right| \int_{0}^{\infty} s^{N}\left|m^{(N+1)}(s)\right| d s .
\end{aligned}
$$

Now take the supremum over $t>0$ on the left hand side, then the $L^{p}$-norm of this inequality to get the assertion by the hypothesis (3.1).

We point out that this simple derivation costs a certain trade-off concerning the size of the parameter $N$. But here we do not care for this aspect.

Similarly to [27] we define the (modified) $K$-functional in the present setting by

$$
K_{\gamma}\left(t, f ; L^{p}, D_{p}\left(D_{c}^{\gamma}\right)\right):=\inf _{g \in D_{p}\left(D_{c}^{\gamma}\right)}\left\{\|f-g\|_{p}+(t(t+c))^{\gamma / 2}\left\|D_{c}^{\gamma} g\right\|_{p}\right\},
$$

where $\gamma>0$ and $c \geq 0$. In essential it is shown in [26] that

$$
\begin{aligned}
K_{\gamma}\left(t, f ; L^{p}, D_{p}\left(D_{c}^{\gamma}\right)\right) & \approx\left\|f-\sum_{k=0}^{\infty} e^{-\phi_{c}^{\gamma}(t) \phi_{c}^{\gamma}(k)} P_{k} f\right\|_{p} \\
& \approx\left\|f-\sum_{k=0}^{\infty}\left(1-\phi_{c}^{\gamma}(t) \phi_{c}^{\gamma}(k)\right)_{+}^{\kappa} P_{k} f\right\|_{p},
\end{aligned}
$$


where $\kappa>N$. Also norm estimates of the de La Vallée Poussin means by the $K$-functional easily follow. Let $m$ be the smooth cut-off function from Example 1.4 (b) (i). Set

$$
V_{\phi ; t} f:=\sum_{k=0}^{\infty} m\left(\phi_{c}^{\gamma}(t) \phi_{c}^{\gamma}(k)\right) P_{k} f, \quad f \in L^{p} .
$$

Then $\left\|V_{\phi ; t} f-f\right\|_{p} \lesssim\|f\|_{p}$ and $\left\|V_{\phi ; t} g-g\right\|_{p} \lesssim \phi_{c}^{\gamma}(t)\left\|D_{c}^{\gamma} g\right\|_{p}$ by Lemma $3.1(\mathrm{~b})$, just as in the $L^{p}\left(\mathbb{R}^{n}\right)$-case. Therefore,

$$
\left\|V_{\phi ; t} f-f\right\|_{p} \lesssim K_{\gamma}\left(t, f ; L^{p}, D_{p}\left(D_{c}^{\gamma}\right)\right), \quad f \in L^{p} .
$$

Thus all ingredients of Sections 1 and 2 are available up to a characterization of the $K$-functional involving moduli of smoothness with respect to generalized translations.

Theorem 3.3 Let $f \in L^{p}, 1<p<\infty$. Choose a positive sequence $\left(\theta_{\ell}\right)_{\ell \in \mathbb{N}_{0}}$ via

$$
K_{\gamma}\left(\theta_{\ell}, f ; L^{p}, D_{p}\left(D_{c}^{\gamma}\right)\right) \equiv K\left(\phi_{c}^{\gamma}\left(\theta_{\ell}\right), f ; L^{p}, D_{p}\left(D_{c}^{\gamma}\right)\right)=2^{-\ell}, \quad 1<p<\infty,
$$

and a decreasing function $F:(0,1) \rightarrow(0, \infty)$ with $\sum_{\ell=0}^{\infty} 1 / F^{p}\left(2^{-\ell}\right)<\infty$. Let $\psi \geq 0$ be a smooth cut-off function with $\psi(t)=1,0 \leq t \leq 1 / 2$, and $=0, t \geq 3 / 4$. Let $h \geq 0$ be a bump function with $\operatorname{supp} h \subset[1 / 2,2]$ such that

$$
\sum_{j=0}^{\infty} h\left(2^{j} \phi_{c}^{\gamma}(t) \phi_{c}^{\gamma}(k)\right)=1 \quad \text { if } \quad 0<\phi_{c}^{\gamma}(t) \phi_{c}^{\gamma}(k)<1 .
$$

Let $m$ be sufficiently smooth and decaying with finite

$$
B_{N}^{*}(m(1-\psi)), \quad B_{N}^{*}\left((1-m) h\left(2^{j} \cdot\right)\right)=: B_{j}^{*}, \quad j \in \mathbb{N}_{0}, \quad N \text { as in (3.1). }
$$

(a) If $t F(t)$ is increasing and

$$
\sum_{j=0}^{\infty} B_{j}^{*} 2^{j}\left(\sum_{\ell: \theta_{\ell}<2^{-j}}^{\infty}\left(\frac{1}{F\left(2^{-\ell-1}\right)}\right)^{p}\right)^{1 / p}<\infty,
$$

then, for every $f \in L^{p}, 1<p<\infty$,

$$
T_{m_{\phi, t}} f(x)-f(x)=o_{x}(1) K_{\gamma}(t, f) F\left(K_{\gamma}(t, f)\right) \quad \text { a. e., } \quad t \rightarrow 0+,
$$

where $o_{x}(1) \in L^{p}\left(\right.$ recall $T_{m_{\phi, t}} f=\sum_{k=0}^{\infty} m\left(\phi_{c}^{\gamma}(t) \phi_{c}^{\gamma}(k) P_{k} f\right.$ on $\left.\mathfrak{P}\right)$.

(b) Let $f$ satisfy $K_{\gamma}(t, f) \lesssim t^{\eta}$ for some fixed $\eta, 0<\eta<1$. If $F(t)=1+$ $|\log t|^{1+1 / p+\varepsilon}, \varepsilon>0$, and $\sum_{j=1}^{\infty}(j+1)^{-1-\varepsilon} B_{j}^{*} 2^{j}<\infty$, then, for every $f \in L^{p}$, $1<p<\infty$, we have

$$
T_{m_{\phi, t}} f(x)-f(x)=o_{x}(1) K_{\gamma}(t, f)\left|\log K_{\gamma}(t, f)\right|^{1+1 / p+\varepsilon} \text { a.e., } \quad t \rightarrow 0+,
$$

where again $o_{x}(1) \in L^{p}$. 
As already suggested above, the proof of Theorem 1.3 carries over without difficulties. Replace $t|\phi|$ there by $\phi_{c}^{\gamma}(t) \phi_{c}^{\gamma}(k)$ here; then the analogs of (2.4) and (2.7) immediately follow. Concerning the critical contribution, analogous to the left term in (2.5), one gets the estimate

$$
\lesssim \sum_{j=0}^{\infty}\left\|T_{((m-1) \psi)_{\phi} h_{2^{j} \phi}}^{*}\right\|_{p \rightarrow p}\left(\sum_{\ell \geq \ell_{0}}\left(\frac{K\left(2^{j} \phi_{c}^{\gamma}\left(\theta_{\ell}\right), f\right)}{K\left(\phi_{c}^{\gamma}\left(\theta_{\ell+1}\right), f\right) F\left(K\left(\phi_{c}^{\gamma}\left(\theta_{\ell+1}\right), f\right)\right.}\right)^{p}\right)^{1 / p}
$$

and can reason as in Section 2 to arrive at the assertions of Theorem 3.3.

\section{Example 3.4. (a) EXPANSIONS INTO SPHERICAL HARMONICS.}

Let $\mathbb{S}^{n}$ be the unit sphere in $\mathbb{R}^{n+1}, n \in \mathbb{N}$, with the origin as center and elements $\xi, \theta, \ldots$, where $\xi=\left(\xi_{1}, \ldots, \xi_{n+1}\right),|\xi|=1$. We denote by $L^{p}\left(\mathbb{S}^{n}\right), 1 \leq p<\infty$, the Banach space of all functions $f(\xi)$ which are $p$-th power integrable on $\mathbb{S}^{n}$, i.e., for which

$$
\|f\|_{p}=\left(\frac{1}{\left|\mathbb{S}^{n}\right|} \int_{\mathbb{S}^{n}}|f(\xi)|^{p} d \sigma(\xi)\right)^{1 / p}<\infty, \quad\left|\mathbb{S}^{n}\right|=\frac{2 \pi^{(n+1) / 2}}{\Gamma((n+1) / 2)},
$$

where $d \sigma$ is the surface element of $\mathbb{S}^{n}$. Let

$$
f(\xi) \sim \sum_{k=0}^{\infty} Y_{k}(f ; \xi), \quad Y_{k}(f ; \xi)=\sum_{m=1}^{H(k, n)} a_{k, m} Y_{k, m}(\xi), \quad f \in L^{p}\left(\mathbb{S}^{n}\right),
$$

be the expansion in a series of spherical harmonics. Here $H(k, n)$ is the number of linear independent spherical harmonics of degree $k$ on $\mathbb{S}^{n}, H(k, n)=(2 k+$ $n-1)(k+n-2) ! /(k !(n-1) !)$. For the Beltrami-Laplace operator $\delta$ one has $\delta Y_{k, m}=-k(k+n-1) Y_{k, m}$, thus set $D_{n-1}^{\gamma}:=(-\delta)^{\gamma}$ - for this setting see, e.g., [28]. The basic property (3.1) with $N>(n-1) / 2$ for spherical harmonics expansions can be found in Bonami and Clerc [3, §3].

Concerning the characterization of the $K$-functional in the present situation, introduce the shift operator $S_{t}$,

$$
\left(S_{t} f\right)(\xi)=\frac{1}{\left|\mathbb{S}^{n-1}\right|(\sin t)^{n-1}} \int_{\xi \cdot \theta=\cos t} f(\theta) d t(\theta), \quad 0<t<\pi,
$$

where $d t(\theta)$ is the element of the section $\{\theta: \xi \cdot \theta=\cos t\}$. The shift operator may equivalently be defined by the generalized translation operator for ultraspherical expansions, since $Y_{k}\left(\left(S_{t} f\right) ; \xi\right)=\left(P_{k}^{(n-1) / 2}(\cos t) / P_{k}^{(n-1) / 2}(1)\right) Y_{k}(f ; \xi)$. If $I$ is the identity operator, introduce a difference operator $\Delta_{t}^{\gamma}$ by $\Delta_{t}^{\gamma}=\left(I-S_{t}\right)^{\gamma / 2}$ and an $\gamma$-th order modulus of smoothness by $[28, \S 4.5]$

$$
\omega_{\gamma, \mathbb{S}^{n}}(t, f)_{p}=\sup _{0<s \leq t}\left\|\Delta_{t}^{\gamma} f\right\|_{L^{p}\left(\mathbb{S}^{n}\right)}, \quad \Delta_{t}^{\gamma}=\sum_{k=0}^{\infty}(-1)^{k}\left(\begin{array}{c}
\gamma / 2 \\
k
\end{array}\right)\left(S_{t}\right)^{k}, \quad \gamma>0 .
$$

Then, by Wang and Li [28, Theorem 5.1.2] (see Kaljabin [12] for $\gamma \in \mathbb{N}$ ),

$$
K_{\gamma}\left(t, f ; L^{p}\left(\mathbb{S}^{n}, D\left(D_{n-1}^{\gamma}\right)\right) \approx \omega_{\gamma, \mathbb{S}^{n}}(t, f)_{p}, \quad 1<p<\infty, \quad \gamma>0 .\right.
$$


Hence we can formulate results analogous to Example 1.4 (b). E.g., in the case of the de La Vallée Poussin means one has for each fixed $L \in \mathbb{N}$

$$
V_{\phi ; t} f(x)-f(x)=o_{x}(1) \omega_{L, \mathbb{S}^{n}}(t, f)_{p}\left|\log \omega_{L, \mathbb{S}^{n}}(t, f)_{p}\right|^{1 / p+\varepsilon} \quad \text { a.e. }, \quad t \rightarrow 0+,
$$

or for the Riesz means $R_{t}^{\kappa, \lambda} f(\xi)=\sum_{k=0}^{\infty}\left(1-\phi_{c}^{\lambda}(t) \phi_{c}^{\lambda}(k)\right)_{+}^{\kappa} Y_{k}(f ; \xi), \kappa>N$, when $0<\gamma<\lambda$

$$
R_{t}^{\kappa, \lambda} f(\xi)-f(\xi)=o_{\xi}(1) \omega_{\gamma, \mathbb{S}^{n}}(t, f)_{p}\left|\log \omega_{\gamma, \mathbb{S}^{n}}(t, f)_{p}\right|^{1 / p+\varepsilon} \quad \text { a.e. }, \quad t \rightarrow 0+.
$$

(Observe that we replace $2^{j} t|\xi|$ of Section 2 here by $2^{j} \phi_{c}^{\gamma}(t) \phi_{c}^{\gamma}(k)$ which leads to $B_{j}^{*} \lesssim 2^{-j \lambda / \gamma}$ in the application of Theorem 3.1 (a).)

For every $f \in L^{p}\left(\mathbb{S}^{n}\right), 1<p<\infty$, with $\omega_{\gamma, \mathbb{S}^{n}}(t, f)_{p} \lesssim \phi_{c}^{\eta}(t)$ for some fixed $\eta$, $0<\eta<\gamma$, we obtain

$$
R_{t}^{\kappa, \gamma} f(\xi)-f(\xi)=o_{\xi}(1) \omega_{\gamma, \mathbb{S}^{n}}(t, f)_{p}\left|\log \omega_{\gamma, \mathbb{S}^{n}}(t, f)_{p}\right|^{1+1 / p+\varepsilon} \quad \text { a. e., } \quad t \rightarrow 0+.
$$

In view of (3.8) and (3.5) a reformulation of this gives a direct estimate of the rate of a.e. pointwise convergence by the rate of strong convergence, i.e., for $t \rightarrow 0+$

$$
R_{t}^{\kappa, \gamma} f(\xi)-f(\xi)=o(1)\left\|R_{t}^{\kappa, \gamma} f-f\right\|_{L^{p}\left(\mathbb{S}^{n}\right)}\left|\log \left\|R_{t}^{\kappa, \gamma} f-f\right\|_{L^{p}\left(\mathbb{S}^{n}\right)}\right|^{1+1 / p+\varepsilon} \text { a. e.. }
$$

This estimate has the advantage that it does not need a characterization of the $K$-functional via a modulus of smoothness based on generalized translations.

We mention that for even integers $2 r, r \in \mathbb{N}$, one can find in [7] the following estimate for the $K$-functional by a different type of modulus of smoothness

$$
K\left(t^{2 r}, f\right) \lesssim \sup _{\rho \in O_{t}}\left\|\Delta_{\rho}^{2 r} f\right\|_{L^{p}\left(\mathbb{S}^{n}\right)}, \quad \Delta_{\rho} f(\xi)=f(\rho \xi)-f(\xi), \quad \Delta_{\rho}^{r}=\Delta_{\rho} \Delta_{\rho}^{r-1} .
$$

Here $\rho \in O_{t}$ are described by those orthogonal matrices with $n \times n$ real entries and determinant 1 , for which $(\rho \theta \cdot \theta) \geq \cos t$ for all $\theta \in \mathbb{S}^{n}$.

(b) Gegenbauer expansions.

For the definitions see, e.g., $[3, \S 6]$ where in particular the crucial property concerning the maximal function of the Cesàro means (3.1) with $N>\lambda+1 / 2$ is proved. For the characterizations (3.4), (3.5), and the estimate (3.6) see [26] $(\alpha=\beta=\lambda-1 / 2)$; here the differential operator is

$$
D=-\left(1-x^{2}\right) \frac{d^{2}}{d x^{2}}+(2 \lambda+1) x \frac{d}{d x} .
$$

For the characterization of the $K$-functional based on generalized translations we refer to $[16]$.

(c) Laguerre And Hermite expansions on $\mathbb{R}_{+}$AND $\mathbb{R}$, resp.

Concerning the basic property (3.1) for Laguerre expansions see [22, p. 126], for Hermite expansions [22, p. 113].

Remark 3.5. (a) Since our intention in Section 3 is only to indicate the method, we don't care for "smallest" admissible $N$ 's (which would have to be 
substituted by some critical $\kappa_{\text {crit }} \in \mathbb{R}_{+}$and would require a fractional calculus cf. [23]).

(b) Estimates for the maximal function of the Cesàro or Riesz means have been established in many settings, in particular in connection with a.e. summability: E.g., for the Fourier-Bessel transform in [20], for compact symmetric spaces of rank 1 in [3, §7], for compact Riemannian manifolds in [9], [10], for some Lie groups in [11].

\section{REFERENCES}

[1] C. Bennett and R. Sharpley, Interpolation of Operators. Academic Press, Boston, 1988.

[2] J. Bergh and J. Löfström, Interpolation Spaces. An Introduction. Springer, Berlin, 1976.

[3] A. Bonami and J.-L. Clerc, Sommes de Cesàro et multiplicateurs de developpements en harmoniques sphériques. Trans. Amer. Math. Soc. 183 (1973), 223-263.

[4] P. L. Butzer, R. J. Nessel, and W. Trebels, On summation processes of Fourier expansions in Banach spaces. I. Tôhoku Math. J. 24 (1972), 127-140; II. ibid. 24 (1972), 551-569.

[5] A. Carbery, Radial Fourier multipliers and associated maximal functions. In: Recent Progress in Fourier Analysis, I.Peral and J.-L. Rubio-de-Francia, Eds., pp. 49 - 56, NorthHolland, Amsterdam, 1985.

[6] H. Dappa and W. Trebels, On maximal functions generated by Fourier multipliers. Ark. Mat. 23 (1985), 241-259.

[7] Z. Ditzian, Jackson-type inequality on the sphere. Acta Math. Hungar. 102 (2004), 1-35.

[8] D. E. Edmunds, P. Gurka, and B. Opic, On embeddings of logarithmic Bessel potential spaces. J. Functional Anal. 146 (1997), 116-150.

[9] L. Hörmander, The spectral function of an elliptic operator. Acta Math. 121 (1968), 193-218.

[10] L. Hörmander, On the Riesz means of spectral functions and eigenfunction expansions for elliptic operators. 1969 Some Recent Advances in the Basic Sciences, Vol. 2 (Proc. Annual Sci. Conf., Belfer Grad. School Sci., Yeshiva Univ., New York, 1965-1966) pp. 155-202.

[11] A. Hulanicki and J. W. Jenkins, Almost everywhere summability on nilmanifolds. Trans. Amer. Math. Soc. 278 (1983), 703-715.

[12] G. A. Kalyabin, On moduli of smoothness of functions given on the sphere. Soviet Math. Dokl. 35 (1987), 619-622.

[13] A. Kamaly, A. Stokolos, and W. Trebels, On the rate of almost everywhere convergence of certain integral means. II. J. Approx. Theory 101 (1999), 240-264.

[14] C. E. Kenig and P. A. Tomas, Maximal operators defined by Fourier multipliers. Studia Math. 68 (1980), 79-83.

[15] K.I. Oskolkov, Approximative properties of summable function on sets of full measure. Mat. Sb. 103 (145), No 4 (1977), 563-589; English transl. in Math. USSR-Sb. 32 (1977), 489-514.

[16] Kh. P. Rustamov, Moduli of smoothness of higher orders related to the Fourier-Jacobi expansion and the approximation of functions by algebraic polynomials. Dokl. Math. 52 (1995), 244-247.

[17] H.S. Shapiro, Smoothing and Approximation of Functions. Van Nostrand Reinhold Co., New York, 1969.

[18] E. M. Stein, Singular Integrals and Differentiability Properties of Functions. Princeton University Press, 1970.

[19] E. M. Stein and G. Weiss, Introduction to Fourier Analysis on Euclidean Spaces. Princeton University Press, 1971. 
[20] K. Stempak, La théorie de Littlewood-Paley pour la transformation de Fourier-Bessel. C.R. Acad. Sci. Paris Sér I Math. 303 (1986), 15-18.

[21] A. Stokolos and W. Trebels, On the rate of almost everywhere convergence of certain integral means. J. Approx. Theory 98 (1999), 203-222.

[22] S. Thangavelu, Lectures on Hermite and Laguerre expansions. Math. Notes, 42. Princeton University Press, 1993.

[23] W. Trebels, Multipliers for $(C, \alpha)$-Bounded Fourier Expansions in Banach Spaces and Approximation Theory. Lect. Notes in Math. 329, Springer, Berlin 1973.

[24] W. Trebels, Some Fourier multiplier criteria and the spherical Bochner-Riesz kernel. Rev. Roumaine Math. Pures Appl. 20 (1975), 1173-1185.

[25] W. Trebels, On the approximation behavior of the Riesz means in $L^{p}\left(\mathbb{R}^{n}\right)$. In: Lect. Notes in Math. 556, pp. 428-438. Springer, Berlin, 1976.

[26] W. Trebels, Equivalence of a $K$-functional with the approximation behavior of some linear means for abstract Fourier series. Proc. Amer. Math. Soc. 127 (1999), 2883 - 2887.

[27] W. Trebels and U. Westphal, On Ulyanov inequalities in Banach spaces and semigroups of linear operators. J. Approx. Theory 160 (2009), 154-170.

[28] K. Wang and L. Li, Harmonic Analysis and Approximation on the Unit Sphere. Science Press, Beijing 2000.

[29] G. Wilmes, On Riesz-type inequalities and K-functionals related to Riesz potentials in $\mathbb{R}^{N}$. Numer. Funct. Anal. Optim. 1 (1979), 57-77.

[30] G. Wilmes, Some inequalities for Riesz potentials of trigonometric polynomials of several variables. In: Proc. Symp. Pure Math. 35, Part 1, pp. 175 - 182, Amer. Math. Soc., Providence, R.I. 1979.

Alexander M. Stokolos

Department of Mathematical Sciences

Georgia Southern University

PO BOX 8093

Statesboro, GA 30460, USA

E-mail address: astokolos@georgiasouthern.edu

AG AGF, Fb. Mathematik

TU DARMSTADT

SCHLOSSGARTENSTR. 7

64289 Darmstadt, Germany

E-mail address: trebels@mathematik.tu-darmstadt.de 